\title{
Beobachtungen der Leoniden 1900
}

an der Sternwarte des Hydrographischen Amtes der k. und k. Kriegsmarine in Pola.

Von den für die diesjährige Beobachtung der Leoniden in Aussicht genommenen fünf Nächten: November 12, 13, 14 , 15 und 16 war leider blos eine, November 13 , durch klares Wetter begünstigt; in den übrigen Nächten bedeckten von Mitternacht an stets dichte Wolken den ganzen Himmel.

November I 3 wachten vier Beobachter von $1 \mathrm{I}^{\mathrm{h}} 3 \mathrm{0}^{\mathrm{m}}$ bis $17^{\mathrm{h}}$ und zählten in dieser Zeit ${ }_{3} 3$ Leoniden und $3^{6}$ andere
Meteore; zur Einzeichnung gelangten blos 8 Meteorbahnen und von diesen gehörten nur 3 (4?) dem Sternbilde des Löwen an. Auffallend sternschnuppenlos blieben gerade die Morgenstunden zwischen $15^{\mathrm{h}} 36^{\mathrm{m}}$ und $17^{\mathrm{h}}$ (ein Meteor).

Folgende $z$ wei Tabellen geben die Details der Beobachtungen; die Numerirung entspricht der zeitlichen Reihenfolge.

I) Verzeichniss der graphisch festgelegten Meteore rgoo Nov. I3.

\begin{tabular}{|c|c|c|c|c|c|c|}
\hline \multirow{2}{*}{$\mathrm{Nr}$. } & \multirow{2}{*}{ M. Z. Pola } & \multicolumn{2}{|c|}{ Anfangspunkt } & \multicolumn{2}{|c|}{ Endpunkt } & \multirow{2}{*}{ Gr. } \\
\hline & & $\alpha$ & $\delta$ & $\alpha$ & $\delta$ & \\
\hline 9 & $12^{\mathrm{h}} 30^{\mathrm{m}} \quad 6^{\mathrm{s}}$ & $10^{h} 12^{m}$ & $+17: 8$ & $10^{\mathrm{h}} 28^{\mathrm{m}}$ & $+16: 9$ & $3-4$ \\
\hline 22 & I3 19 19 & $10 \quad 18$ & +19.8 & I 14 & +19.6 & 2 \\
\hline 23 & $13334^{\circ}$ & 10 $3^{6}$ & +22.0 & 1048 & +21.7 & 3 \\
\hline 25 & I 34 I 58 & 928 & +33.0 & 10 $5^{6}$ & +22.5 & 4 \\
\hline
\end{tabular}

\begin{tabular}{|c|c|c|c|c|c|c|c|}
\hline \multirow{2}{*}{$\frac{\mathrm{Nr}}{28}$} & M. Z. Pola & \multicolumn{2}{|c|}{ Anfangspunkt } & \multicolumn{3}{|c|}{ Endpunkt } & Gr. \\
\hline & $14^{\mathrm{h}} 0^{\mathrm{In}} 9^{\mathrm{s}}$ & $7^{h} 5^{6^{m}}$ & $+3: 0$ & & $49^{\mathrm{m}}$ & $-3: 9$ & 2 \\
\hline 33 & 142742 & 617 & +30.7 & 4 & 20 & +10.5 & Sirius \\
\hline 46 & $\begin{array}{lll}15 & 31 & 34\end{array}$ & 1050 & +33.8 & 12 & 44 & +54.7 & 2 \\
\hline 48 & $15 \quad 5434$ & & -10.5 & & 4 & -9.5 & 2 \\
\hline
\end{tabular}

Nr. 9, 22,23 (?), 46 Leoniden, die übrigen fremd.

II) Verzeichniss der übrigen nicht graphisch festgelegten Meteore rgoo Nov. r3.

\begin{tabular}{|c|c|c|c|c|c|c|c|c|c|c|}
\hline $\mathrm{Nr}$. & M. Z. Pola & Gr. & Spur & Anmerkung & Nr. & M. Z.P & Pola & Gr. & Spur & Anmerkung \\
\hline I & $1^{h} 5^{6^{m}}$ & - & ohne & Aus Perseus & 26 & $13^{\mathrm{h}} 47^{\mathrm{n}}$ & & 2 & ohne & $\varepsilon$ Leonis $-\gamma$ Leonis \\
\hline 2 & 1158 & 2 & hell & Leonide & 27 & 1347 & & $1-2$ & & $\alpha$ Lyrae-iUrsae majoris \\
\hline 3 & I 25 & $\mathbf{I}$ & hell & Aus Gemini & 29 & I 4 I & $14^{\mathrm{s}}$ & 3 & ohne & Aus Orion \\
\hline 4 & 1210 & 2 & hell & Aus Gemini & 30 & $14 \mathrm{I}$ & 38 & $1-2$ & $1^{s}$ & Aus Gemini \\
\hline 5 & 1210 & - & - & Aus Taurus & $3 \mathrm{r}$ & 145 & 19 & 2 & - & Fremd \\
\hline 6 & $121559^{5}$ & - & - & Aus Taurus & $3^{2}$ & 148 & 59 & 3 & - & Aus Gemini \\
\hline 7 & 12163 & $\mathbf{I}$ & hell & Leonide & 34 & 1436 & 59 & $3-4$ & schwach & Leonide \\
\hline 8 & 1226 & $\mathbf{I}$ & keine & Aus Perseus & 35 & 1437 & 24 & $3-4$ & schwach & Leonide \\
\hline ro & $12 \quad 34 \quad 19$ & 4 & keine & Aus Taurus & $3^{6}$ & 1442 & 17 & 2 & schwach & Leonide \\
\hline ( I 1) & $1237 \quad 54$ & 2 & schwach & Aus Taurus & $\left.37^{2}\right)$ & I 48 & $4 \mathrm{I}$ & $I-2$ & hell & Aus Lynx-Canes venat. \\
\hline$\left(2^{1}\right)$ & $1238 \quad 54$ & 2 & schwach & Aus Taurus & $3^{8}$ & I 45 I & 54 & - & - & Fremd \\
\hline 13 & 12440 & 2 & keine & Fremd & 39 & $145^{2}$ & 9 & I & hell $2^{5}$ & $\alpha$ Lyrae $-\lambda$ Draconis \\
\hline I 4 & 1248 & - & - & Fremd & 40 & 159 & 29 & $2-3$ & schwach & Aus Canis major \\
\hline I 5 & 1250 & - & - & Aus Gemini & $4 I$ & 1514 & I 9 & 4 & ohne & Fremd \\
\hline 16 & I $254 \quad 54$ & 3 & schwach & Aus Orion & 42 & 1524 & 14 & $3-4$ & ohne & Fremd \\
\hline I 7 & $1256 \quad 14$ & $1-2$ & hell & Leonide & 43 & $15 \quad 25$ & 54 & $3-4$ & ohne & Fremd \\
\hline I 8 & I $257 \quad 34$ & $3-4$ & - & Aus Orion & $\left.44^{3}\right)$ & 1527 & 35 & 1 & ohne & Leonide \\
\hline I 9 & I $3 \quad$ I 39 & $\mathbf{I}-\mathbf{2}$ & schwach & Aus Gemini & 45 & 1529 & & $3-4$ & schwach & Fremd \\
\hline 20 & 13840 & $1-2$ & hell & Aus Taurus & 47 & I 55 & 54 & 3 & ohne & Fremd \\
\hline $2 \mathbf{I}$ & 131029 & $\mathbf{I}$ & ohne & Aus Gemini & 49 & 1615 & 44 & $1-2$ & ohne & Fremd \\
\hline 24 & I3 368 & 3 & schwach & Leonide & & & & & & \\
\hline
\end{tabular}

1) Beide Bahnen parallel.

2) Auffallend roth.

3) Weiss, zerplatzt nach 5 Secunden Dauer.

November 14, gelegentlich einer nur kurz währenden Aufheiterung, sah Herr Linienschiffsfähnrich $R$. Höhl um $6^{\mathrm{h}} 35^{\mathrm{m}}$ ein Meteor $1-2 \mathrm{Gr}$. von $14^{\mathrm{h}} 8^{\mathrm{m}}+54^{\circ} 4^{2}$ nach $12^{\mathrm{h}} 4^{\mathrm{m}}+58^{\mathrm{O}} 2$ fliegen, mit einer 2 Secunden andauernden Spur.
Beobachter waren die Herren:

K. und k. Linienschiffsfähnrich E. Marchetti

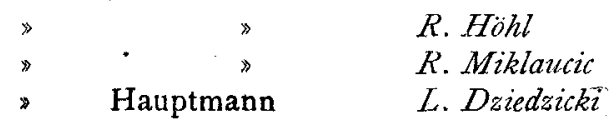

Der Vorstand: I. von Benko.

Pola, 1900 November. 